$\frac{197 / 28 / 71}{197}$

\section{The Gyrncon: A Deflection-Modulated,} High-Power Microwave Amplifier

Paul J. Tallerico

\section{UC-28}

Issued: October 1977 


\title{
THE GYROCON: A DEFLECTION-MODULATED, HIGH-POWER MICROWAVE AMPLIFIER
}

by

Paul J. Tallerico

\begin{abstract}
A iarge-signal, relativistic theory of the electron-field interaction in a new class of microwave amplifiers is presented and applied to the analysis of a high-power, $450-\mathrm{MHz}$ amplifier for accelerator applications. The analysis indicates that electronic effrciencies in excess of $90 \%$ are obtainable and that overall efficiencies of $90 \%$ are possible. The amplifier is unique in several respects; the electron velocity is perpendicular to the circuit energy flow, the device uses a fast-wave circuit, and the electron beam is deflection modulated.

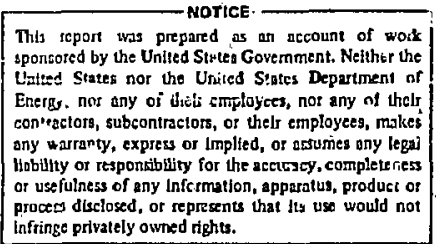

\section{INTRODUCTION}

A novel electron device, called a gyrocon or bermutron* and capable of functioning as a highly afficient, rory high power microwave amplifier, oscillator, or frequency multiplier, is analyzed in this report. The gyrocon, invented in $1946,{ }^{1}$ has only recently been shown ${ }^{2}$ capable of producing very high output power and excellent dc-to-rf conversion efficiency. Such an efficierst energy converter makes practical several types of large, high-power rf systems. It can reduce drastically the construction and operating costs of very high power accelerators, especially those that call for high-energy, high-beam currents, or a high duty factor. Th? feasibility of high-current, $\mathrm{cw}$ accelerators for producing fissile material muy depend on amplifier properties.

The gyrocon operates by deflection, rather than velocity modulation, of an electron beam. Because the deflection is produced by the magnetic fields of a microwave cavity, operation at very high beam voltage is possible. The basic device, shown in Fig. 1, comprises an electron gun, a deflection cavity, an output resonator, a collector, and an output coupler. The electron gun produces a cylindrical, solid electron beam. The deflection cavity must support two modes with magnetic fields perpendicular to the beam direction. When the two component fields are $90^{\circ}$ out of phase in time and space, the resultant field rotates, exactly like the field in a two-phase electric motor. The rotating deflection field causes the beam to travel in an expanding helical path, although the envelope of the trajectories is conical. The beam enters a slot in the outpat resonator, a resonant ring of rectangular waveguide. The phase velocity of the waveguide fie!d is greater than

\footnotetext{
"Russian workers use the name "gyrocon." Gyrocon implies gyrating constantly, the feature of the device that they consider essential. "Bermutron," an acrorym for beam-rotating multiplier, has been used in American literature. We use gyrocon here because the device is more efficient as an amplifier than as a multiplier.
} 
the velocity of light, c, but the deflection velocity of the beam, ulso a phase velucity, can be synchronized with the field. The collector is the back of the weveguide. A suitable output coupler extracts the microwave power from the output resonator. With the proper choice of output resonator geometry and rf voltage, the electron beam can be almost $t_{2}$ alted in transit across the waveguide. Thus, the dc-to-rf conversion efficiency can be very high. The waveguide field is the dominent $\mathrm{TE}_{01}$ mode, and the electrons are stopped by the electric field. The electrons need not be bunchsad, because each part of the electron beam experiences the same fields. The deflection cavity may operate at a subharmonic $n$ of the output frequency if the output resorator is $n$ wavelengths long.

The basic device was patented in 1946 by J. W. McRae to generate high frequencies by frequency multiplication. Kaufman and Oltman, ${ }^{3}$ in 1965 , built a frequency multipliei that generated $0.4 \mathrm{~W}$ of power at $34 \mathrm{GHz}$ with a $65 . \mathrm{W}$ drive power at $8.5 \mathrm{GHz}$. Their device had a linear output cavity, a length of shorted rectangular guide. Their paper conteins several references to unpublished work on similar devices in the $1950 \mathrm{~s}$. In 1958, P. B. Wilson at Stanford investigated a very large family of deflection modulation devices, including ihe gyrocon, theoretically, but no devices were built.

Recently, G. I. Budker ${ }^{2}$ at the Institute for Nuclear Research in No osibirsk has shown visitors a 1.3$\mathrm{MV}, 40-\mathrm{A}$ gyrocon that is alleged to prcduce $40 \mathrm{MW}$ of of power at $430 \mathrm{MHz}$. Budker's improvement in the basic device is a bending magnet, shown in Fig. 2, that bends the beam into the output waveguide. The bending magnet greatly reduces the deflection power needed to modulate the beem, because the deflection power is proporcional to the square of the tangent of the deflection angle.

This report develops the first-order theory for the dc-to-rf conversion efficiency $\bar{\eta}$ of the gyrocon. The theory alsc is useful for designing gyrocons. Most of the equations have been programmed into GYRO, an analygis code used to invegtigate the effec:s of beam voltage, current, and frequency on gyrocon performance.

In the gyrocon, it is very easy to obtain an overall $\bar{\eta}$ of 80 to $90 \%$, whereas in the klystron, fo: example, the calculated $\bar{\eta}$ is usually 50 to $70 \%$. Tnus, accelerator applications that are impractical usirig conirentional if sources may bo practical with the gyrocon. The power per of source may be increased suostantiolly because

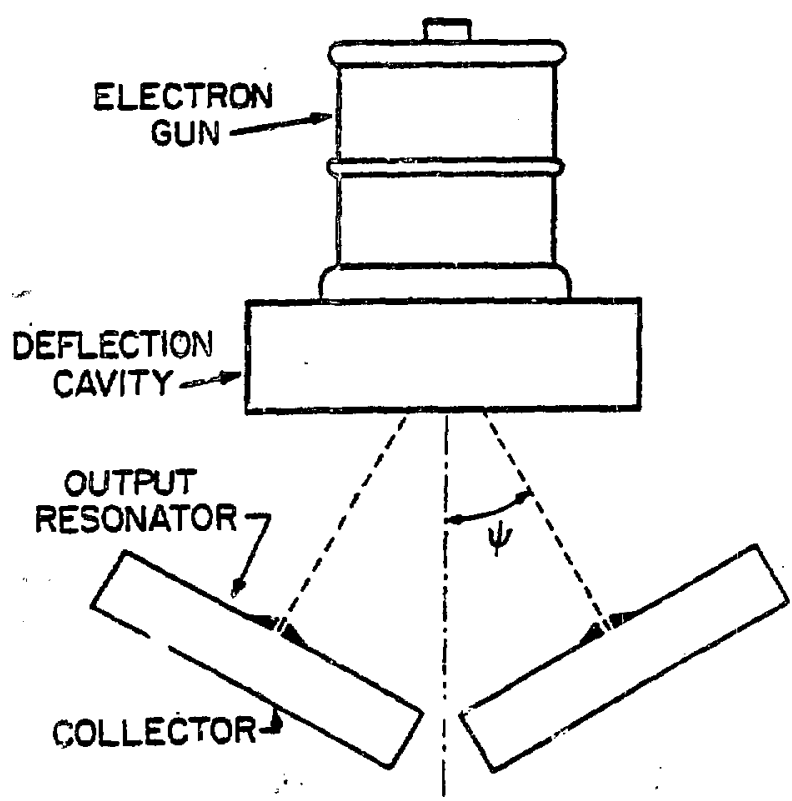

Fig. 1.

The basic gyrocon.

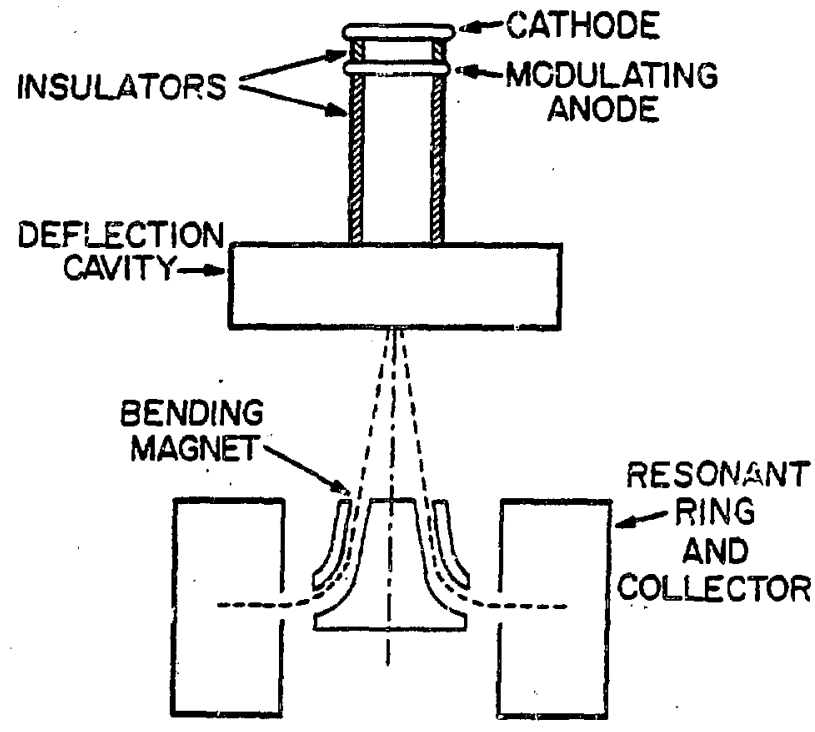

Fig. 2.

The improved gyroson with a bending magnet to reduce deflection power. 
of the large output circuit and excellent efficiency of the gyrocon. Although the power gain of a gyrocon is much smaller than that of a klystron or a traveling-wave tube, the possibility of high output power and high efficiency justifies further analysis and experimentation.

For analytical purposes, it is convenient to write $\bar{y}$ as

$$
\bar{\eta}=\bar{\eta}_{0} \bar{\eta}_{\mathrm{c}} \bar{\eta}_{\mathrm{a}} \bar{\eta}_{t}
$$

where $\eta_{0}=$ the electronic conversion efficiency

$\eta_{c}=$ the output circuit efficiency

$\eta_{\mathrm{d}}=$ the deflection circuit efficiency

$\eta_{t}=$ the focusing and bending aystem efficiency.

Each term in Eq. (1) can be calculated independently, and, then, the overall efficiency calculated.

First, the output interaction and circuit are analyzed to establish the electronic and circuit efficiencies. Next, the deflection circuit is analyzed. The bending system is discussed only in general terms because the author has not yet analyzed this part of the gyrocon. The trajectory from the electron gun to the output circuit is analyzed, and the report conclucies with several specific design examples that illustrate the performance of gyrocon amplifiers as functions of current, voltage, and frequency.

\section{OUTPUT INTERACTION}

\section{A. First-Order Analysis}

The basic principle of the gyrocon is the injection of an unbunched electron beam into a resonant ring of $\mathrm{TE}_{01}$-mode waveguide. If the conditions are proper, the beam will be hulted as it travels across the waveguide, and the dc-to-rf conversion efficiency will be very high. A highly idealized case with the following assumptions clarifies the problem.

1. The electric field $E_{y}$ in the output circuit parallel to the beam direction provides the only aignifi-

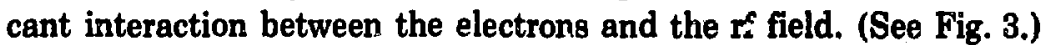

2. The electron beam enters the waveguide at $x=a / 2, y=b$ with velocicy $u_{0}$ in the minus $y$ direction. The beam is so small that it is considered infinitely thin.

3. The effects of space charge are negligible.

4. The dc beam velocity $u_{0}$ is so small that relativistic effects are neglected.

5. The waveguide field results from a straight, uniform, rectangular waveguide; that is: vuuputwaveguide curvature effects are neglected.

6. All higher order modes in the waveguide are neglected.

These assumptions define tine ideal gyrocon. An ancilysis of a more general case in which the first four restrictions are removed is presented later, but the ideal case has simple equations that can be integrated easily. Without loss is generality, the z-axis origin is placed in the output section at a traveling-wave minimum, at $t=0$. The beam is injected at $z_{\mathrm{z}}=\xi / \beta$, and $\mathrm{t}=0$, as shown in. Fig. 4. The waveguide dimensions, electric field, and power losses are calculated next for the ideal case.

The electric field in the waveguide is

$$
E_{y}=-E_{0} \cos (\omega t-\beta z)
$$

where $\omega$ is the radian frequency, $\beta$ is the propagation constant of the wave, and $E_{0}$ is the field amplitude. The force equation is 


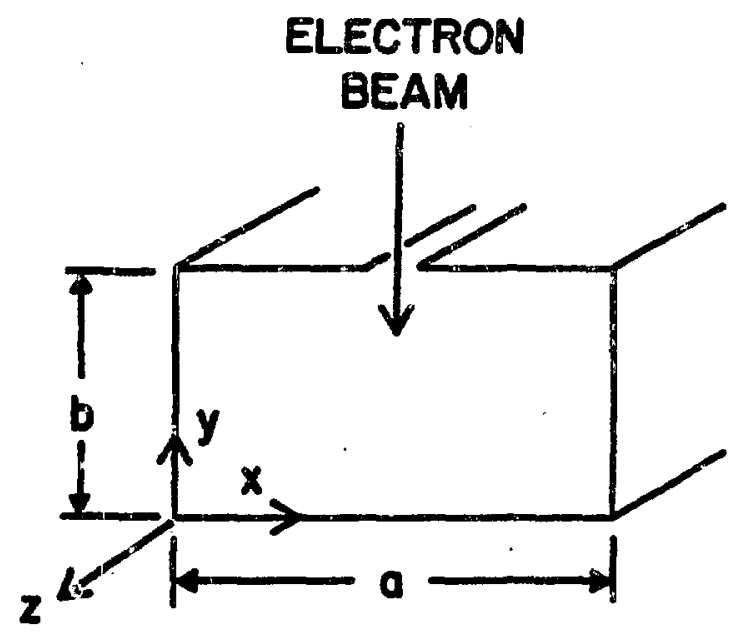

Fig. 3.

The output interaction region.

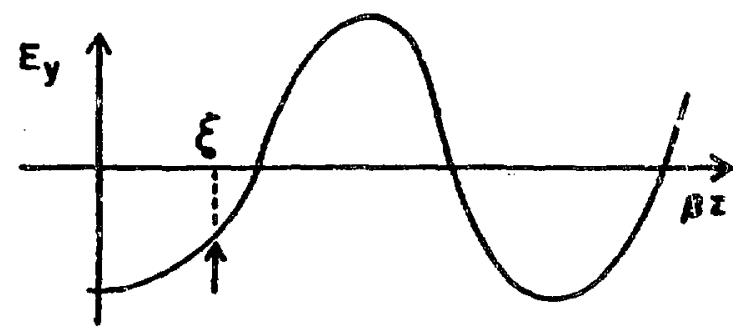

Fig. 4.

The electric field in the output waveguide at $t=0$.

$$
\frac{d v_{y}}{d t}=n E_{0} \cos (\omega t-\phi)
$$

where $\eta=|e| / m_{0}$ and $\phi=\beta z$. The electrons in the ideal case have no motion in the $z$ - or $z$-directions. Equation (3) may be integrated to determine the veiocity;

$$
v_{y}(t)=-\theta_{0}+\frac{\eta E_{0}}{\omega}[\sin (\omega t-\phi)+\sin \phi]
$$

The velocity snay be integrated to find the position;

$$
\left.y(t)=b-\mid u_{0}-\frac{1 \varepsilon_{0}}{\omega} \sin \phi\right) t-\frac{n E_{0}}{\omega^{2}}[\cos (\omega t-\phi)-\cos \phi] .
$$

From Eq. (4), the velocity is zero when

$$
E_{0}=\frac{\omega u_{0}}{\pi[\sin (\omega t-\phi)+\sin \phi]}
$$

The lowest loss solution will have the minimum $\mathrm{E}_{5}, 80$ from Eq. (6) the optimum transit time r and entry phase $\phi$ are

$$
\omega \gamma=(2 m+1) \pi, \quad m=0,1,2 \ldots,
$$

and

$$
\phi=\pi / 2 .
$$


The condition $y(\tau)=0$ yields

$$
b=\left(u_{0}-\frac{n E_{0}}{\omega} \sin \phi\right) \tau+\frac{n E_{0}}{\omega^{2}}[\cos (\omega \tau-\phi)-\cos \phi] .
$$

Putting the optimum values from Eq. (7) into Eqs. (4) and (8) produces

$$
E_{0} \quad \frac{w u_{0}}{2 n}
$$

and

$$
b=\pi u_{0} /(2 u)
$$

wheze the $m=0$ trajectory is chosen as the minimum loss case. The rf voltage in the output waveguide is

$$
v_{r f}=E_{0} b=\frac{\pi}{2} V_{0}
$$

where $V_{0}$ is the dc beam voltage that corresponds to $u_{0}$; that is, $V_{0}=u_{0}^{2} /(2 \eta)$. All the beam's kinetic energy is converted to electric energy in the waveguide; thus, $\eta_{e}=1.0$. In Sec. I.C, large-signal equations for determining $\eta_{0}$ more accurately are derived.

\section{B. Output-Circuit Effieiency}

The output circuit is a resonant ring of waveguide. For simplicity, consider a rectangular waveguide bent in a circle, shown in Fig. 5 in cross section. Because the fieids in the waveguide are $\mathrm{TE}_{01}$, the mode can be described ${ }^{0}$ as

$$
\begin{aligned}
& E_{y}=E_{0} \sin \frac{\pi x}{a} \cos (\omega t-B z) \\
& H_{x}=\frac{E_{0}}{Z_{T E}} \sin \frac{\pi x}{a} \cos (\omega t-\beta z) .
\end{aligned}
$$

and

$$
H_{z}=\frac{E_{0}}{n_{0}} \frac{\lambda}{2 a} \cos \frac{\pi x}{a} \sin (\omega t-\beta z),
$$

where $\lambda=c / f, c$ is the velocity of light in vacuum, $\eta_{0}=\left(\mu_{0} / \epsilon_{0}\right)^{1 / 2}$, 
$Z_{Y E}=n_{0}\left[1-\left(\frac{\lambda}{2 a}\right)^{2}\right]^{-\frac{1}{2}}$, and $\beta=\frac{2 \pi}{\lambda_{g}}$, where $\lambda_{g}=\lambda\left[1-\left(\frac{\lambda}{2 a}\right)^{2}\right]^{-\frac{1}{2}}$.

The circuit efficiency is defined as

$$
n_{c}=1-\frac{P_{L}}{P_{T}}
$$

where $P_{T}$ is the power transmitted in the waveguide and $P_{L}$ is the power dissipated in the waveguide walls. The transmitted power is

$$
\begin{aligned}
P_{T} & =\frac{1}{2} s_{0}^{b} j_{0}^{a} E_{y} H_{x} d x d y \\
& =\left[1-\left(\frac{\lambda}{2 a}\right)^{2}\right]^{\frac{1}{2}} E_{0}^{2} a b /\left(4 n_{0}\right)
\end{aligned}
$$

The resonant ring is designed so that

$$
2 \pi r_{\tilde{a}}=n \lambda_{8}, \quad n=1,2 \ldots .
$$

A bit of algebra shows the power lost in the waveguide walls to be

$$
P_{L}=\frac{n \pi\left(1+\frac{b \lambda^{2}}{2 a^{3}}\right) a E_{0}^{2}}{n_{0}\left(2 \sigma \mu_{0} \nu\right)^{\frac{1}{2}}\left[1-\left(\frac{\lambda}{2 a}\right)^{2}\right]^{\frac{1}{2}}} \text {, }
$$

where $\sigma$ is the condlictivity of the waveguide material. The overall circuit efficiency can be obtained by evaluating Eqs. (16) and (18) and placing the results in Eq. (15). The parameter $n$ should be set to 1 for greatest efficiency, unless $x_{\mathfrak{g}}$ from Eq. (17) must be increased. At high frequencies, the circuit efficiency decreases because the losses are proportional to $\omega^{1 / 2}$.

\section{Output-Section Electron Dymamics}

The relativistic force equation is

$$
\frac{d}{d t}(\gamma \vec{v})=-\eta\left(\vec{E}+\mu_{0} \vec{v} \times \vec{H}\right)
$$


where $\quad \gamma=\left(3-\frac{v_{x}^{2}+v_{y}^{2}+v_{z}^{2}}{c^{2}}\right)^{-\frac{1}{2}}$.

After expansion of the force equation with the relation

$$
\frac{d y}{d t}=-\frac{\eta}{c^{2}} v_{y} E_{y}
$$

and the fields of Eqs. (12)-(14), the component equationg are

$$
\begin{aligned}
& \frac{d v_{y}}{d t}=-\frac{n}{\gamma}\left\{E_{y}\left[1-\left(\frac{v_{y}}{c}\right)^{2}\right]+\mu_{0}\left(v_{z} H_{x}-v_{x} H_{z}\right)\right], \\
& \frac{d v}{d t}=-\frac{\eta v}{\gamma}\left(\mu_{0} H_{z}-\frac{v_{x} E_{y}}{c^{2}}\right),
\end{aligned}
$$

and

$$
\frac{d v_{z}}{d t}=\frac{n v_{y}}{r}\left(\frac{v_{z} E_{y}}{c^{2}}+\mu_{0} H_{x}\right) \text {. }
$$

A second approximation of the electronic efficiency (see Sec. II.A) is obtsined by integrating Eqs. (21)(24) through the output waveguide, for $\mathrm{a}$ given set of fields. The beam is divided into one central core and $\mathbf{N}_{\mathrm{R}}$ rirgs; each ring is subdivided inico $\mathrm{N}_{\mathrm{T}}$ segments, as shown in Fig. 6 . The first-order effect of space charge is calculated by allowing the potential, hence the $y$-directed beam velocity, to vary with the beam radius. Each beamlet in Fig. 6 contains an equal amount of current. If the beam has current $I_{0}$ and an initial radius $r_{0}$, the potential at any radius $r<r_{0}$ is

$$
v(r)=v_{0}+\frac{I_{0} r^{2}}{4 \pi u_{0} r_{0}^{2} \varepsilon_{0}}
$$

where $V_{0}$ is the potential on the axis and $I_{0}$ is positive. The numerical integration procedure is checked by calculating $\gamma$, the energy factor ior each electron, by using Eqs. (20) and (21). The results would be identical if the integration were perfect. The difference between the two calculated energies usually is $<2 \%$.

\section{Other Output Wavezuide Considerationg}

The presence of the slot shown in Fig. 5 increases the waveguide wavelength by 


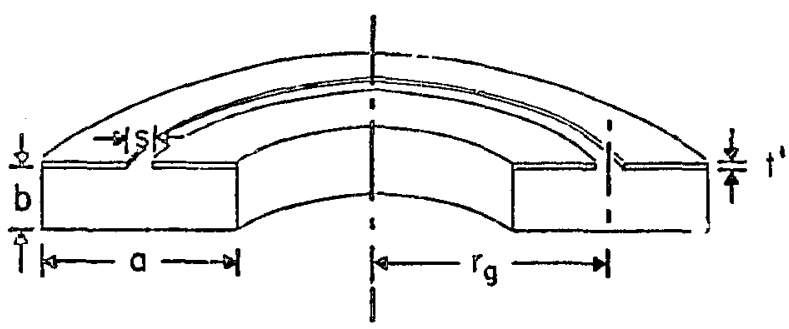

Fig. 5.

The circular output waveguide.
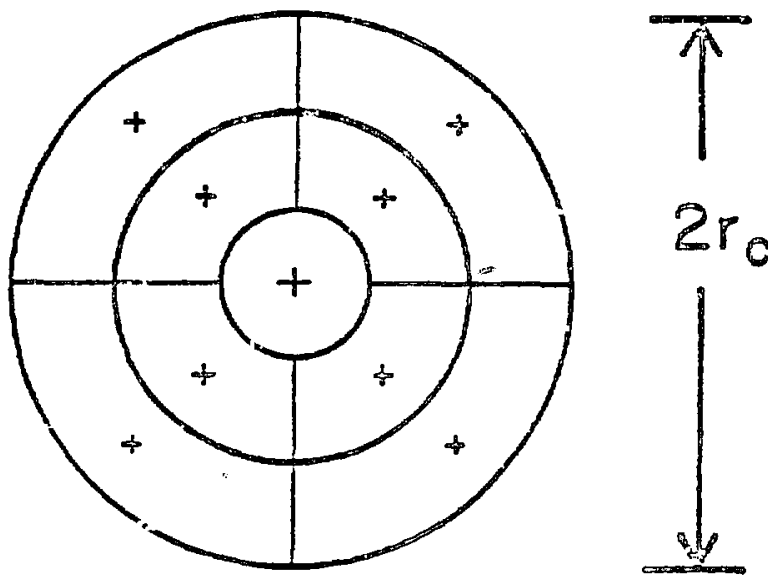

Fig. 6.

Division of the beam into $N_{\mathrm{H}}$ rings and $N_{\mathrm{T}}$ sutdivisions. Here $N_{\mathrm{R}}=3$ and $N_{\mathrm{T}}=4$.

$$
\frac{\Delta \lambda_{g}}{\lambda_{g}} \approx \frac{\pi s^{2}}{16 a b}\left(\frac{\lambda_{g}}{2 a}\right)^{2} \quad \text { if } t^{\prime} \approx 0
$$

or

$$
\frac{\Delta \lambda_{g}}{\lambda_{g}} \approx \frac{s^{2}}{2 \pi a b}\left(\frac{\lambda_{g}}{2 a}\right)^{2} \quad \text { i i } t^{\prime} \gg s
$$

In each case, it is assumed that $\mathrm{s} / \mathrm{b} \ll 1$. Thus, in a typical case, $r_{\mathrm{g}}$ is a few percentage points larger than Eq. (17) indicates. The slot radiates no energy to first order.

A major omission in the equations presented in Sec. $\mathrm{C}$ is the effect of space charge on the beam within the output waveguide. As the bean is almost stopped in the output region, space-charge forces are large as the beamlets land on the collector. These low-energy electrons generate secondary electrons that can extract energy from the output fields.

The output coupler is not considered here. Russian reports ${ }^{2}$ indicate that two couplers are needed; one probably extracts the power and the other probably attenuates the wave that rotates in the wrong direction. Wilson' has shown how to build a simple two-aperture coupler to extract the energy from a gyrocon. The apertures may be on either surface of the output circuit, but it seems prudent to locate them in the narrow wall, farthest from the electrons. An ov'sput coupler with many apertures will work better then a coupler with only two ancertures because the cutput circuit voltage is more constant with angle in the former.

\section{THE DEPLECTION CAVITY}

Microwave cavities have been used to deflect electron beams in several accelerators. Panofsky and Wenzel ${ }^{7}$ analyzed the problem in general terms and showed that TM modes will work, whereas TE modes 
produce no net transverse deflection because the electric and magnetic forces cancel each other at any beam velocity. Haimson ${ }^{8}$ has studied the problem using aeveral modes in rectangular and cylindrical cavities. His analysis is fairly complete, even to tracing rays through the deflection fields to find the aberration.

In this section the deflection produced by a $\mathbf{T M}_{103}$ field in a rectangular deflectin $\mathrm{n}$ cavity is analyzed. Then the power required to support these fields is calculated. Some of Haimson's aberration results are discussed and applied to this problem.

\section{A. Deflection Angle and Fields}

The $\mathrm{TM}_{108}$ mode seems best for a gyrocon deflection cavity. Either rectangular or cylindrical cavities can be used, but only the rectangular one is considered here. Figure 7 show/s the cavity and its coordinate system. The beam is injected parallel to the $y$-axis at $z=a_{1} / 2, x=b_{1} / 2$, and we assutme that the beam entrance and exit, apertures do not affect the cavity fields.

The electric field, in the y-direction, is

$$
E_{y}=E_{0} \sin \frac{2 \pi z}{a_{1}} \sin \frac{\pi x}{b_{1}}
$$

where $E_{0}$ is an arbitrary constant. The magnetic field components are

$$
H_{2}=\frac{j \omega E_{0} E_{i} \pi}{b_{1} k_{c}^{2}} \sin \frac{2 \pi z}{a_{1}} \cos \frac{j \pi x}{b_{1}}
$$

and

$$
H_{x}=-\frac{j \omega E_{0} E_{0} 2 \pi}{a_{j} k_{c}^{2}} \cos \frac{2 \pi z}{a_{1}} \sin \frac{\pi x}{b_{1}},
$$

where

$$
k_{c}^{2}=\left(\frac{2 \pi}{a_{1}}\right)^{2}+\left(\frac{\pi}{b_{1}}\right)^{2}
$$

and the resonant wavelength is

$$
\lambda=2 \pi / k_{c} .
$$

The very attractive feature of this mode is that, for a thin electron beam, $E_{y}=H_{z}=0$ at $z=a_{2} / 2$,

Fig. 7.

The $T M_{102}$ deflection cavity. 
$x=b_{i} / 2$. Thus, there is no velocity modulation to first order for a small-diameter beam. Therefore, the transverse force equation is

$$
\frac{d\left(\gamma_{0} v_{z}\right)}{d t}=-\frac{2 \pi m w d_{0}}{a_{1} k_{c}^{2} c^{2}} \sin (\omega t+\phi)
$$

where $u_{0}$ is the axial velocity and $\gamma_{0}$ is the mass factor. With a small deflection angle, the beam passes through no electric field, so $\gamma_{0}$ is constant.In Eq. (30), $\phi$ is an arbitrary phase of the magnetic field relative to the entrance time of the particles being deflected. Equation (30) may be integrated to yield

$$
v_{z}(y=d)=\frac{2 m u_{0} E_{0}}{\gamma_{0} a_{0} \omega^{2}}[\cos (D+\phi)-\cos \phi],
$$

where $D=\omega d / u_{0}$, the transit angle. Maximum deflection occurs when $\phi=(\pi-D) / 2+n \pi$, and the deflection velocity then is

$$
v_{z}=\frac{4 m E_{0} u_{0}}{\gamma_{0} a_{1} \omega^{2}} \sin D / 2
$$

The deflection angle is $\psi$, where

$$
\tan \psi=\frac{v_{2}}{u_{0}}=\frac{4 \pi m E_{0}}{\gamma_{0} a_{1} \omega^{2}} \sin D / 2 .
$$

\section{B. Power Loss in Cavity Walls}

The surface currents on the cavity. walls are given by

$$
\vec{J}=\hat{\mathbf{n}} \times \overrightarrow{\mathbf{H}} \quad \because
$$

where $n$ is the unit normal to the cavity surface and the power dissipated in the walls is

$$
P_{C}=\frac{R_{S}}{2} \int S|\vec{J}|^{2} d S
$$

: where $S$ is the surface of the cavity and $R$ is the skin-effect resistance,

$$
\mathbf{R}_{0}=\left[\omega \mu_{0} /(2 \sigma)\right]^{1 / 2}
$$


After considerable algebi. ., Eqs. (34)-(36) yield

$$
P_{0}=\frac{E_{0}^{2} E_{0}^{2} \omega^{2} R_{s_{1}} a_{1}^{4}}{\pi^{2}\left[4+\left(\frac{a_{1}}{b_{1}}\right)^{2}\right]^{2}}\left(\frac{a_{1}}{4 b_{1}}+\frac{b_{1}}{a_{1}}+\frac{2 b_{1}^{d}}{a_{1}^{2}}+\frac{d a_{1}}{2 b_{1}^{2}}\right) .
$$

To achieve a rotating deflection, another mode, $90^{\circ}$ out of time and space phase, must be added: Therefore, $a_{1}$ must be equal to $b_{1}$, and $P_{o}$ must be doubled. Then Eqs. (37) and (29) become

$$
P_{0}=\frac{R_{S}\left|E_{0} a_{1}^{2} \omega E_{0}\right|^{2}}{10 \pi^{2}}\left[1+\frac{2 d}{a_{1}}\right]
$$

and

$$
\lambda=\frac{2 a_{1}}{\sqrt{5}}=0.89443 a_{1} .
$$

\section{Optimum Length of Deflection Cavity}

Equation (39) determines the dimension $a_{1}$ of the cavity, and it is convenient to combine Eqs. (33), (36), and (38) to obtain

$$
P_{0}=c_{v} \gamma_{0}^{2} f^{\frac{1}{2}} \frac{\tan ^{2} \psi^{2}}{\sin ^{2}\left(\frac{\pi f d}{u_{0}}\right)}\left(1+\frac{4 d f}{c \sqrt{5}}\right) .
$$

where

$$
c_{1}=\frac{125 \pi^{2} c^{5} \varepsilon_{0}^{1.5}}{160 \sigma^{1 / 2} n^{2}}=2.0862 \text { for copper. }
$$

The deflection power varies with the square root of frequency and with the square of the defiection-angle tengent. For any given deflection angle, beam voltage, deflection length, and frsquency, we can use Eq. (40) to calculate the deflection power. However, there is an optimum cavity length, found by differentiating Eq. (40) and satting the result equal to zero. The author has not been able to solve the resulting transcendental equation exactly, but 


$$
d_{\mathrm{opt}} \approx \frac{u_{0}}{4 r}\left[1-a+\sqrt{(1+\alpha)^{2}}-\frac{8}{\pi^{2}}\right] .
$$

where

$$
\alpha=\frac{c \sqrt{5}}{2 u_{0}}
$$

is an approximate solution. The quantities $\gamma_{0}$ and $u_{0}$ are found from

$$
\gamma_{0}=I+\frac{\eta v_{0}}{c^{2}}
$$

and

$$
u_{0}=c\left(1-1 / \gamma_{0}^{2}\right)^{-\frac{1}{2}}
$$

where $V_{0}$ is the beam voltage on the axis.

\section{Aberrationo and Neglected Quantities in the Deflection Cavity}

Hairason has integrat ad the force equations through a $\mathrm{TM}_{102}$ deflection cavity and a $0.2-\mathrm{m}$ drift space. He neglected space-charge and aperture effects and found few aberrations for a beam size of $0.1 \lambda$, whereas for $0.2 \lambda$, the beam was highly elli ${ }^{-}$-ical. The required force equations are Eqs. (21)-(24), but Eqs. (12) to (14) must be used for the fields. Thus, uYRO, which integrates Eqs. (21)-(24), can be modified to integrate the deflection-cavity equation. This work has not yet been done. The effects of space charge in the drift space beyond the deflection cavity are calculated approximately below.

\section{THE BENDING SYSTEM}

Budker's improvement to the gyrocon, the bending magnet shown in Fig. $z$, is a major achievement because it reduces the required deflection angle by a factor of about 5 . This redices the required deflection power by the square of the factor. In many cases, the gyrocon power gain is a factor of only 10 to 15 , and an additional 10 to 25 makes the device much more attractive.

The author has not analyzed the bending aystem, but he makes the following points. There are at least four methods, illustrated in Fig. 8 , that can be used for a bending system. The first-order system uses $\varepsilon$ current sheet; it has the advantage that the magnetic field can be perpendicular to the electron velocity. There is a serious problem where the current sheet crosses the beam path, but the deflection is proportional to the current. The second-order bending system has a simple solenoid within the cone of the beam trajectories, and it is easier to build, but the deflection is proportional to the square of the current, sc many more 


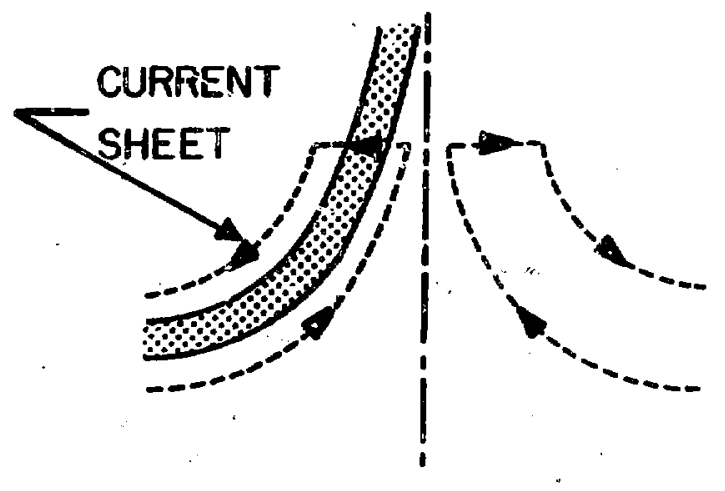

a) FIRST ORDER

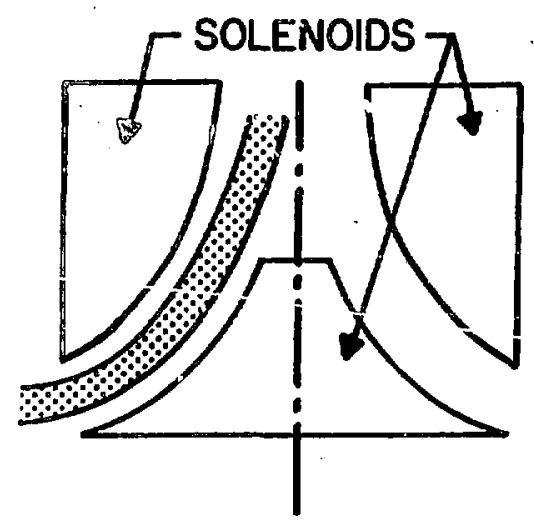

c) MAGNETIC CUSP

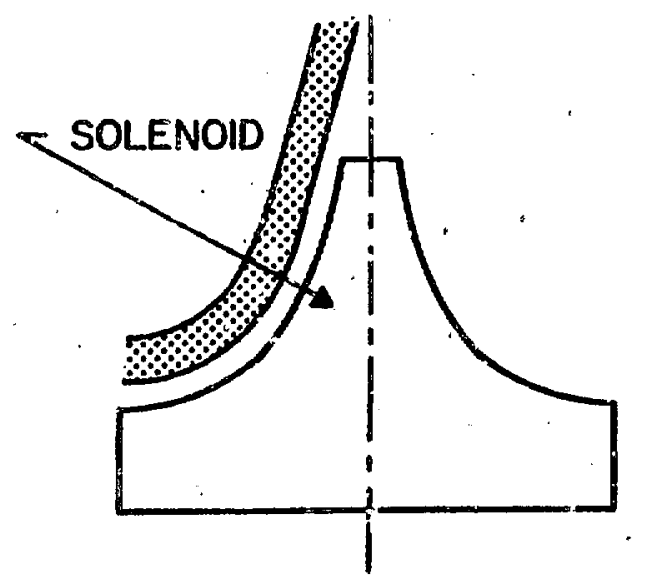

b) SECOND CRDER

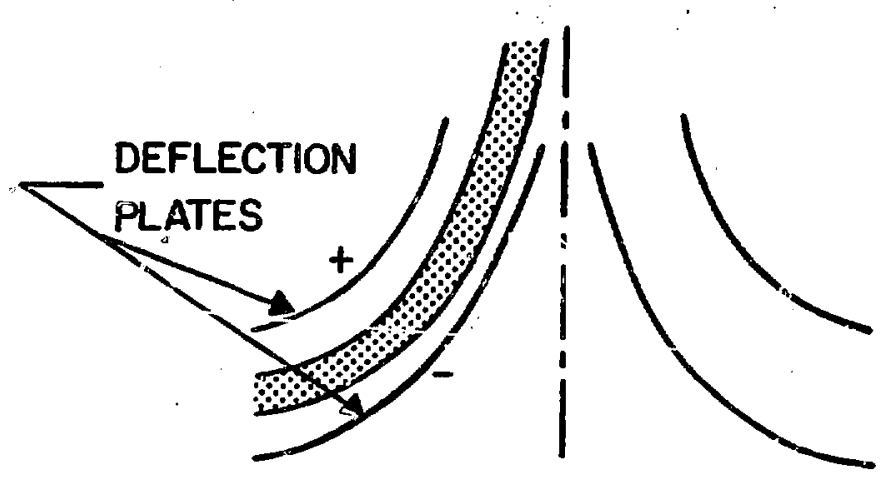

d) EL_ECTROSTATIC

Fig. 8.

Four possible beam-bending systems.

ampere turns are required. Miller has pointed out that there must be an of imum current-sheet shape for deflection or aberration characteristics, but that shape will be a challenge to find. The magnetic cusp system has two solenoids whose fields oppose each other; it works like a magnetic mirror and defiects the beam outward. It also is a second-order system; it requires even more ampere turns because the fields partially cancel each other. For a high-power, high-voltage gyrocon, a magnetic bending system is best, but for lower power models, it may be possible to use an electrostatic bending system. In each case, the detailed optics must be studied carefully. The focusing prope:ties of each bending system are important in selection of the bending system.

\section{SPACE-CHARGE EFFECTS}

The major space-charge effect outsidie the output cavity is to force the beam to grow as it travels. The waveguide slot must be kept as small as possible, but the beam must not touch the slot edges. Thus, the beam size must be estimated. 
The zadial force equation is ${ }^{10}$

$$
r \frac{d v_{r}}{d \frac{x}{c}}=-n E_{r}\left[1-\left(\frac{v_{r}}{c}\right)^{2}\right\}_{j}+n y_{2}\left(B_{\theta}+\frac{v_{r}}{c^{2}} E_{2}\right)+\frac{r \omega_{c}^{2}}{4 \gamma}\left[1-k\left(\frac{r_{0}}{r}\right)^{4}\right],
$$

where $\mathrm{K}$ is the cathode flux parame $\mathrm{e}^{4}$ :

$$
k=\left(\frac{r_{c}}{r_{0}}\right)^{4}\left(\frac{B_{c}}{g_{0}}\right)^{2}
$$

The subscripts $c$ and $o$ refer to quantities evaluated at the cathode and the magnetic field entrance region, respectively, and $\omega_{c}=\eta \mathrm{B}_{a}$. We assume that the flow is leminar and that both the radial and azimuthal velocities are small compared to the velocity of light. The axial velocity is constant at 11., end the force equation can be transformed to

$$
\frac{d^{2} r}{d z^{2}}=\frac{n I_{0}}{2 \pi \varepsilon_{0} u_{0}^{3} \gamma_{0}^{3} r}-\frac{\omega_{c}^{2}}{4 \gamma_{0}^{2} u_{0}^{2}}\left(r-K \frac{r_{0}^{4}}{r^{3}}\right)
$$

where we have used the itatic fields

$$
\begin{aligned}
& E_{r}=\frac{I_{0}}{2 \pi r E_{0} \dot{H}_{0}}, \\
& B_{\theta}=\frac{\mu_{0} I_{0}}{2 \pi r},
\end{aligned}
$$

and

$$
E_{2}=0
$$

We assume that Eq. (47) holds true even though the central trajectory is not straight, and the beam may not aven be circular because of aberration from the deflection cavity. Equation (47) has been integrated for several configuracions, as shown in Fig. 9. There may be a magnetic lens at the beginning of the trajectory computation, and, beyond the lens, the waynetic field is zero. Figure 10 shows the trajectories for a LAMPF klystron gun operating at $86 \mathrm{kV}$ and $11.6 \mathrm{~A}$ with $\mathrm{B}_{\mathrm{z}}=0,100$, and $200 \mathrm{G}$. Trajectories for higher voltages and no magnetic lenses are shown in Fig. 11. The beam spread is much less at $200 \mathrm{kV}$, the highest voltage considered. 


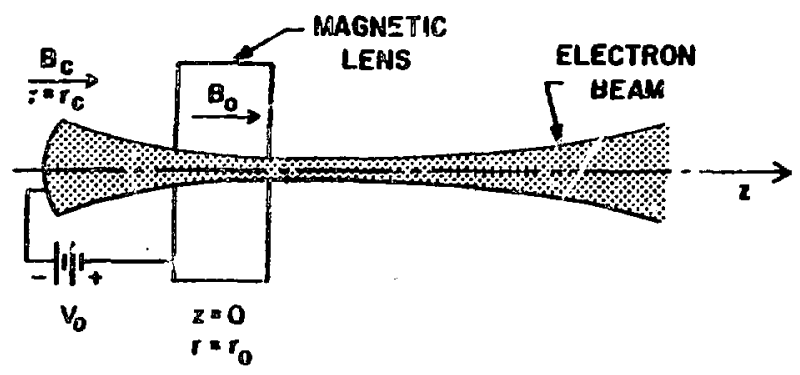

Fig. 9.

The model used for beam-spiead calculations.

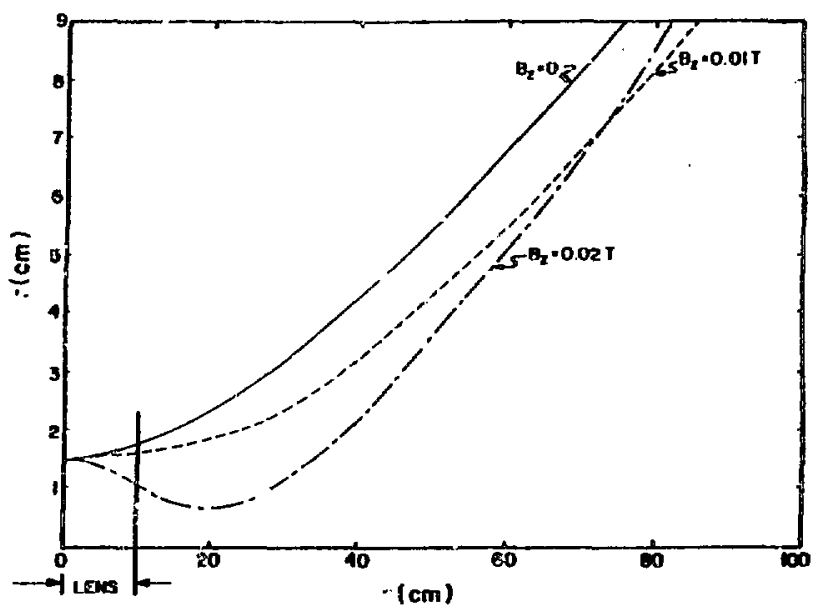

Fig. 10.

Beam spread of the LAMPF klystron gun with a 10-cm-long magnetic lens.

\section{GYROCON DESIGN EXAMPLAS}

Most of the preceding equations have been assembled into the FORTRAN computer code GYRO. Using GYRO, one can get most first-order design parameters for any gyrocon amplifier quickly. The bending system equations are not included, so only the simple device shown in Fig. 1 can be analyzed. The primary design consideration is that, at any dc beam voltage $V_{0}$, the rf voltage in the output waveguide must be approsin: ately $\pi V_{d} / 2$ to stop the electrons and give high conversion efficiency. With such a waveguide voltage, power losses in the output waveguide will be tens of kilowatta; therefore, fur high efficiency, beam power mugt he hundreds of hiluwatis. $\bar{\nu}$ 'tlection power is independent of beam power because beam loading has been neglected; cutput power must be large for high deflection efriciency and power gain.

The first design, shown in Fig. 12(a), has a $30^{\circ}$ deflection angle and operates at $450 \mathrm{MHz}$ with no bending system, and the distance from the center of the deflection cavity (the beam minimum) to the entrance of the output waveguide is $40 \mathrm{~cm}$. The second design, shown in Fig. 12(b), has a $10^{\circ}$ deflection angle and operates at $450 \mathrm{MHz}$, and the distance from the center of the deflectirn cavity to the entrance of the output waveguide is $110 \mathrm{~cm}$. In each design, the waveguide radius is $19.15 \mathrm{~cm}$, or a few percentage points greater, to account for the slot effects. No focus lens was used, and the minimum beam radius provided by the electron gun was assumed to be $1.5 \mathrm{~cm}$, about that of the beam from the LAMPF klystron at $86 \mathrm{kV}$.

First, \& matrix of calculations on the besm size at the output waveguide was made for these initial conditiors as a function of beam voltage and current. The results are shown in Table 1 . The step size used to integrate Eq. (47) was $0.5 \mathrm{~cm}$, and the cathode flux parameter was set to zero. The slot width in the output waveguide must be as small as possible, but it should be about $40 \%$ larger than the beam diameter. With a wayeguide width of $40 \mathrm{~cm}$, a beam radius of $<4 \mathrm{~cm}$ gives a slot width of $<11 \mathrm{~cm}$. If the slot width is nearly equal to the waveguide height, Eq. (26) will not be valid for estimating the guide wavelength increase due to the slot. A more important problem could be radiation from the slot. Both problems should be attacked by an experimental program, and both are eliminated by operating the device at a dc voltage of several hundred kilovolts or higher. At $20 \mathrm{kV}$, the beam is too large at any power level, and at $50 \mathrm{kV}$, the amplifier can operate only at $100 \mathrm{KW}$ input. Thus, dc beam-spread calculations alone show that high output power gyrocons toquire high dc beam voltages. 


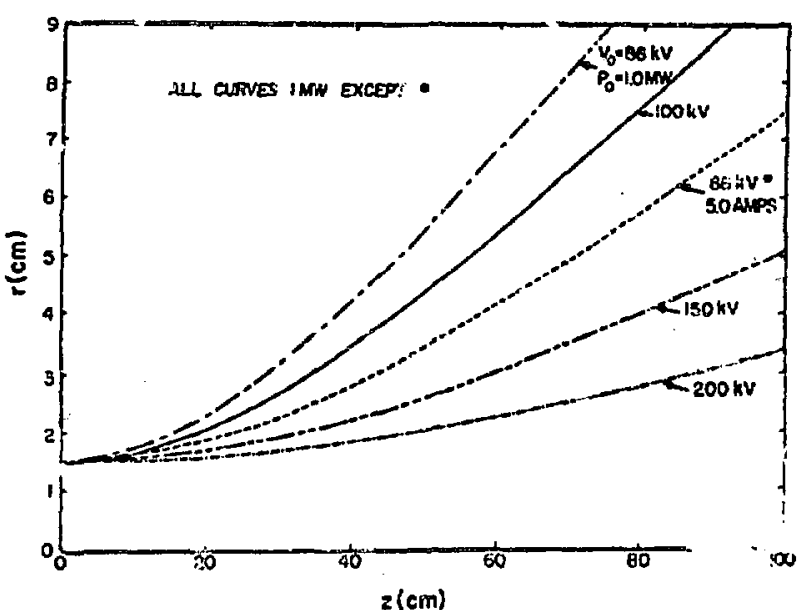

Fis. 11

LAMPF klystron gun trajectories as a function of beam voltage with no magnetic lens.

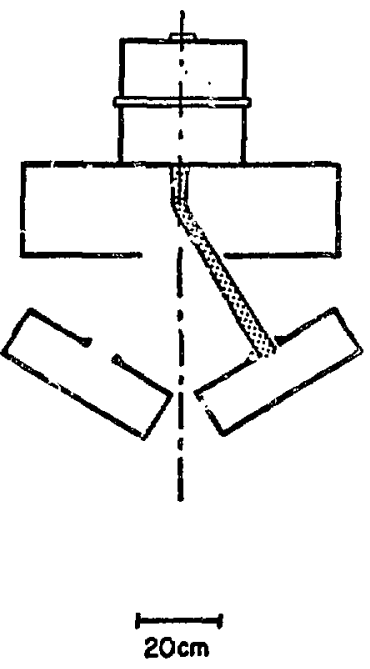

(a)

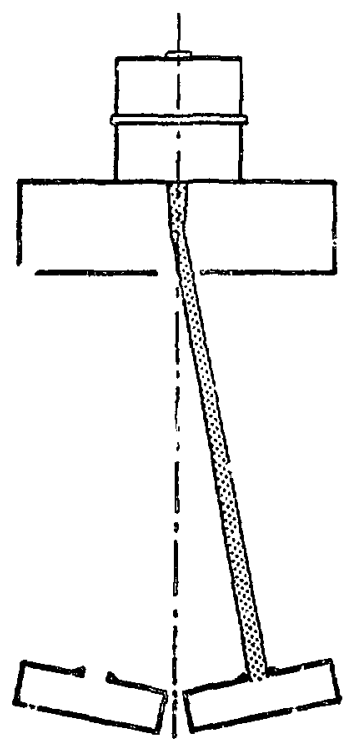

(b)

Fig. 12.

Scale drawizgs of two gyrocons for $450-\mathrm{MHz}$ :

(a) $30^{\circ}$ deflection; (b) $10^{\circ}$ dejlection.

Next, tue deflection system garameters were calculated. The sạuare deflection cavity ig $74.5 \mathrm{~cm}$ nn a side and resonates at $450 \mathrm{MHz}$. The optimum cavity length and the power required to deflect the beam hy 10 and $30^{\circ}$ are listed in Table II. Estimates of the Jeflection power are optimistic, because beam-loading effects heve been neglectes. The $30^{\circ}$ deflection system becomes impractisal at voltages above $500 \mathrm{kV}$, but the $10^{\circ}$ system is practical up to $2 \mathrm{M} \checkmark$.

TABLE I

BEAM RADIUS AT THE OUTPUT WAVEGUIDE VERSUS BEAM :R

$r(40 \mathrm{~cm})$

$r(40 \mathrm{~cm})$

$\begin{array}{rrrrrr}\begin{array}{c}V_{0} \\ (\mathrm{kV})\end{array} & \begin{array}{c}P_{0}=700 \\ (\mathrm{kH})\end{array} & & \begin{array}{l}300 \\ (\mathrm{~kW})\end{array} & \begin{array}{r}1000 \\ (\mathrm{~kW})\end{array} & \begin{array}{r}3000 \\ (\mathrm{~kW})\end{array} \\ 20 & 9.90 & 20.00 & 42.90 & 83.60 \\ 50 & 2.71 & 4.66 & 9.71 & 19.60 \\ 100 & 7.72 & 2.13 & 3.40 & 6.26 \\ 200 & 1.53 & 1.60 & 1.84 & 2.46 \\ 500 & 1.50 & 1.51 & 1.53 & 1.58 \\ 1000 & 1.50 & 1.50 & 1.50 & 1.51 \\ 2000 & 1.50 & 1.50 & 1.50 & 1.50\end{array}$

$r(.10 \mathrm{~cm})$

\begin{tabular}{cccc}
\hline 100 & 300 & 1000 & 3000 \\
$\frac{(k H)}{(k W)}$ & $\frac{(k W)}{(k W)}$ & $\frac{1}{(k W)}$ \\
\hline 35.50 & 66.60 & 154.00 & 273.00 \\
8.09 & 16.40 & 35.1 & 69.20 \\
2.97 & 5.28 & 11.1 & 22.50 \\
1.76 & 2.23 & 3.68 & 6.90 \\
1.52 & 1.56 & 1.69 & 2.04 \\
1.50 & 1.51 & 1.52 & 1.56 \\
1.50 & 1.50 & 1.50 & 1.51
\end{tabular}


The most time-consuming part of the analysis was integration of Egs. (21)-(24) with the central core and. th s four beamlets spaced equally around it. The if fields used in these equations are from Eqs. (12)-(14) ex$c \in p i$ that the time and the z-variations are now relative to an entrance phase angle, $\xi$.

For each combination of waveguide field $E_{0}$ and waveguide height $b$, the entrance phase angle was awept in $5^{\circ}$ steps to find the highest energy conversion efficiency. The time step was $2^{\circ}$ of the $450-\mathrm{MHz}$ if fields, and the beam radius at the output-v:aveguide entrance was from Table I. Calculations were made for the cases in which this radius was smaller than $20 \mathrm{~cm}$, half the output waveguide width. Enough runs were made to optimize $E_{0}$ to $\pm 5 \%$ and $b$ to $\pm 1 \mathrm{~cm}$. The values of $b$ under $10 \mathrm{~cm}$ were optimized to $\pm 1 / 2 . \mathrm{cm}$. A very nice feature is that the electronic efficiency varies very slowly with $b$ and $E_{0}$. Changes in $b$ of 2 to $4 \mathrm{~cm}$ and in $E_{0}$ of $10 \%$ change the optimum value of the entrance phase significantly, but change the electronic efficiency by only 1 or $2 \%$. The optimum values found to date are listed in Table III for the $30^{\circ}$ system and in Table IV for the $10^{\circ}$ system. At the optimum entrance phase, the electrons are not stopped in the injection direction because they gain energy. rapidly in the z-direction as $y$ decreases. The optimum condition gives equa: amounts of enargy in the $y$ - and $z$-directions. The $z$-directed velocity comes from the magnetic force on the electrons in the waveguide; this force was neglected in the one-dimensional results. . he de beam power for the three sets of results are 100,300 , and $1000 \mathrm{~kW}$. Higher power devices could be brilt, but the $1-\mathrm{MW}$ veam input limit demonstrates the potential of the gyrocon.

The electronic efficiency of the $30^{\circ}$ syrtem (Table III) reaches a maximum of about $95 \%$ at $100 \mathrm{kV}$ at each beam power, and it is independent of the beam current. If space-charge effects can.be included more accurately, the beam current will have a stronger effect on the electronic eff' ciency. The overall efficiency, the total rf output power divided by the beam input plus the deflection power, also is shown in Table III. Usually, overall efficiencies increase as dc beam power increases, because waveguide losses depend on beam voltage, nct on beam power. At $2 \mathrm{MV}$, at least. $2 \mathrm{MW}$ of power is ' $x$ st in the waveguide, so a gyrocon must be very ligh powered to be efficient at $2 \mathrm{MV}$. Budker's design, ${ }^{2}$ with a $100-\mathrm{MW}$ beam power could be very efficient, as only $2 \%$ of the beam power is lost in the waveguide. The rf power gain (Table III) also increases with beam power, because the deflection power depends on beam voltage, not on beam nower: The rf power gain for the $10^{\circ}$ device (Taile IV) is about $10 \mathrm{~dB}$ higher in each case; but the electronic efficiency is lower because the beam is larger when it reaches the output waveguide.

Calculations were macie for a six-times frequency multiplier with a $30^{\circ}$ deflection system. A 200-KV beam voltage and a 5-A current were assumed. Figure 12(a) applies, except that the waveguide broad dimen. sion is now $6.67 \mathrm{~cm}$. The beam radius is still $1.84 \mathrm{~cm}$, because the diameter of the output guide has not

TABLE II

OPTIMUM DEFLECTION CAVITY LENGTH, FIELD AMD POWER VERSUS BEAM VOLTAGE

\begin{tabular}{rrccc}
$\begin{array}{c}V_{0} \\
(\mathrm{kV})\end{array}$ & $\begin{array}{c}\mathrm{d} \\
(\mathrm{cm})\end{array}$ & $\begin{array}{c}E_{0} \\
(\mathrm{MV} / \mathrm{m})\end{array}$ & $\begin{array}{l}P_{0}, 30^{\circ} \\
(\mathrm{kW})\end{array}$ & $\begin{array}{r}P_{0^{\prime}}, 10^{\circ} \\
(\mathrm{kW})\end{array}$ \\
\hline 20 & 8.5 & 1.62 & 17.52 & 1.64 \\
50 & 13.0 & 1.71 & 21.44 & 2.01 \\
100 & 17.0 & 1.87 & 27.58 & 2.58 \\
200 & 21.0 & 2.19 & 40.50 & 3.78 \\
500 & 27.0 & 3.11 & 89.00 & 8.32 \\
1000 & 27.0 & 4.67 & 207.10 & 19.32 \\
2000 & 30.0 & 7.76 & 579.80 & 54.08
\end{tabular}


TABLE III

$30^{\circ}$ OGFLECTION-ANGLE aMPLIFIER SUMARY

\begin{tabular}{|c|c|c|c|c|c|c|c|c|c|c|}
\hline $\begin{array}{c}v_{0} \\
(k v) \\
\end{array}$ & $\begin{array}{r}I_{0} \\
(A) \\
\end{array}$ & $\begin{array}{c}r_{0} \\
(\mathrm{~cm})\end{array}$ & $\stackrel{t}{(\mathrm{~cm})}$ & $\begin{array}{c}E_{0} \\
(m y / m)\end{array}$ & $\begin{array}{c}E \\
\text { (deg) }\end{array}$ & $\begin{array}{l}\text { Ele_tronic } \\
\text { Ef: iciency }\end{array}$ & $\begin{array}{c}P_{L} \\
(k W)\end{array}$ & $\begin{array}{l}P_{\text {out }} \\
\text { (kU) }\end{array}$ & $\begin{array}{l}\text { Gain } \\
\text { (dBI }\end{array}$ & $\begin{array}{r}\text { Overal } \\
\text { Efficien }\end{array}$ \\
\hline & \multicolumn{10}{|c|}{ 100-kw dc Beam Power } \\
\hline 20 & 5.00 & 9.90 & 3.0 & 0.80 & 40 & 0.9242 & 6.6 & 85.8 & 17.2 & 0.7303 \\
\hline 50 & 2.00 & 2.71 & 8.0 & 1.00 & 95 & 0.9574 & 12.0 & 83.8 & 16.9 & 0.6895 \\
\hline 100 & 1.00 & 1.72 & 10.0 & 1.50 & $=90$ & 0.9587 & 28.4 & 67.5 & 3.9 & 0.5288 \\
\hline 200 & 0.50 & 1.53 & 10.6 & 2.40 & 70 & 0.9328 & 72.7 & 20.6 & -0.3 & 0.1463 \\
\hline 500 & 0.20 & 1.50 & 14.0 & 4.20 & 75 & 0.8755 & 245.6 & - & . & - \\
\hline 1000 & 0.10 & 1.50 & 14.0 & 7.00 & 55 & 0.8134 & 682.3 & - & - & - \\
\hline 2000 & 0.05 & 1.50 & 10.5 & 16.00 & 35 & 0.7435 & 3231.9 & - & - & - \\
\hline \multicolumn{11}{|c|}{ juO-kW dc Beam Power } \\
\hline 20 & 15.00 & 20.00 & 3.0 & 0.90 & 30 & 0.6736 & 8.4 & 193.7 & 20.8 & 0.6100 \\
\hline 50 & 6.00 & 4.66 & 5.0 & 1.15 & 50 & 0.9455 & 14.6 & 269.1 & 22.0 & 0.8370 \\
\hline 100 & 3.00 & 2.13 & 9.0 & 1.50 & 80 & 0.9516 & 27.7 & 257.8 & 9.7 & 0.7890 \\
\hline 200 & 1.50 & 1.73 & 12.0 & 2.20 & 80 & 0.9299 & 64.3 & 214.7 & 7.2 & 0.6306 \\
\hline 500 & 0.60 & 1.50 & 16.0 & 4.00 & 85 & 0.8689 & 233.2 & 27.5 & -5.1 & 0.0706 \\
\hline 1000 & 0.30 & 1.50 & 20.0 & 5.40 & 95 & 0.8069 & 650.2 & - & - & - \\
\hline 2000 & 0.15 & 1.50 & 10.0 & 12.00 & 70 & 0.7496 & 2098.7 & - & - & - \\
\hline \multicolumn{11}{|c|}{ 1000-kH dc Beam Power } \\
\hline so & 20.00 & 9.70 & 5.0 & 1.20 & 40 & 0.9154 & $? 3$ & 899.6 & 27.2 & 0.8807 \\
\hline 100 & 10.00 & 3.40 & 7.0 & 1.70 & 60 & 0.9509 & 33.7 & 917.2 & 15.2 & 0.8926 \\
\hline 200 & 5.00 & 1.84 & 12.0 & 2.20 & 80 & 0.9333 & 64.3 & 869.1 & 13.3 & 0.8353 \\
\hline 500 & 2.00 & 1.53 & 16.0 & 4.00 & 85 & 0.8765 & 233.2 & 543.3 & 8.6 & 0.5907 \\
\hline 1000 & 1.00 & 1.50 & 18.0 & 6.50 & 85 & $0 . \div 33$ & 643.2 & 170.1 & -0.9 & 0.1409 \\
\hline 2001 & 0.50 & 1.50 & 16.0 & 12.00 & 70 & 0.7496 & 2098.7 & - & - & - \\
\hline
\end{tabular}

changed. The best electronic efficiency $(86.5 \%$ ) occurred prith a $1.2-\mathrm{cm}$ waveguide height and an $18.0-\mathrm{MV} / \mathrm{m}$ field. The circuit losses exceed the rf power at this high field, so the device cannot operate. A 2.4-cm-high waveguide gave an optimum overall efficiency of $29.1 \%$, with a $6-\mathrm{MV} / \mathrm{m}$ field in the output guide. The overall efficiency is this low because the waveguide fields are this high; thus, the waveguide losses are high. A smallor initial beam diameter and operation as a straight amplifier would raise the overall efficiency, but whether this type of amplifier can compete with a well-designed klystron at operating frequencies above $3 \mathrm{GHz}$ is doubtful. A reentrant output circuit, such as a ridge-loaded guiae, would probably improve the efficiency at these higher frequencies. When analyzed previously" for low-efficiency operation at very high frequencies, the device showed little promise.

\section{SUMMARY AND CONCLUSIONS}

A class of deflection-modulated, high-power amplifiers has been analyzed. The large-signal calculations indicate that overall efficiencies approaching $90 \%$ and 10 - to 30 -dB power gains are achievable in the 450 $\mathrm{MHz}$ region. Design parameters such as the electron gun and waveguide width were not varied to achieve the 
TABLE IV

10 DEFLECTION-ANGLE AMPLIFIER SUMMRRY

\begin{tabular}{|c|c|c|c|c|c|c|c|c|c|c|}
\hline $\begin{array}{c}V_{0} \\
(k V) \\
\end{array}$ & $\begin{array}{r}I_{0} \\
(A) \\
\end{array}$ & $\begin{array}{c}r_{0} \\
(\mathrm{~cm}) \\
\end{array}$ & $\stackrel{b}{\mathrm{~cm} l}$ & $\begin{array}{c}E_{0} \\
(N) / m)\end{array}$ & $\begin{array}{c}E \\
\text { (deg) }\end{array}$ & $\begin{array}{l}\text { Electronic } \\
\text { Efficiency } \\
\end{array}$ & $\begin{array}{c}P_{L} \\
(k W)\end{array}$ & $\begin{array}{l}P_{\text {out }} \\
(k \text { kW) }\end{array}$ & $\begin{array}{l}\text { Gain } \\
(B)\end{array}$ & $\begin{array}{c}\text { Overall } \\
\text { Efficiency }\end{array}$ \\
\hline & \multicolumn{10}{|c|}{ 100-kW dc Beam Power } \\
\hline 50 & 2.00 & 8.09 & 5.0 & 1.20 & 45 & 0.9402 & 15.8 & 78.2 & 26.9 & 0.7664 \\
\hline 100 & 1.00 & 2.97 & 7.0 & 1.65 & 55 & 0.9556 & 31.7 & 63.8 & 13.9 & 0.6223 \\
\hline 200 & 0.50 & 1.76 & 11.0 & 2.30 & 75 & 0.9349 & 68.5 & 25.0 & $8.2^{\circ}$ & 0.2408 \\
\hline 500 & 0.20 & 1.52 & 16.0 & 4.00 & 85 & 0.8771 & 233.2 & - & - & - \\
\hline 1000 & .0 .10 & 1.50 & 14.0 & 7.60 & 55 & 0.8134 & 682.3 & - & - & - \\
\hline \multirow[t]{2}{*}{2000} & 0.05 & 1.50 & 10.0 & 16.00 & 35 & $0.7 / 435$ & 3221,9 & - & - & - \\
\hline & \multicolumn{10}{|c|}{ 300-kH dc Beam Power } \\
\hline 50 & 6.00 & 16.40 & 4.0 & 1.50 & 25 & 0.7958 & 24.0 & 214.7 & $31: 3$ & 0.7110 \\
\hline 100 & 3.00 & 5.28 & 6.0 & 1.85 & 45 & 0.9459 & 38.8 & 245.0 & 19.8 & 0.8097 \\
\hline 200 & 1.50 & 2.23 & 9.0 & 2.50 & 55 & 0.9348 & 76.9 & 203.6 & 17.3 & 0.6702 \\
\hline 500 & 0.60 & 1.56 & 16.0 & 4.10 & 85 & 0.8764 & .233 .2 & 8.9 & 10.3 & 0.0286 \\
\hline 1000 & 0.30 & 1.51 & 16.0 & 6.60 & 70 & 0.8146 & 634.8 & - & - & - \\
\hline \multirow[t]{2}{*}{2000} & 0.15 & $: \quad 1.50$ & 16.0 & $12.0 \mathrm{C}$ & 70 & 0.7496 & 2098.7 & - & $=$ & - \\
\hline & & & & \multicolumn{4}{|c|}{ 1000-kW dc Beam Power } & & & \\
\hline 100 & 10.00 & 11.10 & 7.0 & 1.75 & 45 & 0.8899 & 35.7 & 854.2 & 25.2 & 0.8520 \\
\hline 200 & 5.00 & 3.68 & 8.0 & 2.70 & 45 & 0.9307 & 87.3 & 843.4 & 23.5 & 0.8402 \\
\hline 500 & 2.00 & 1.69 & 14.0 & 4.10 . & 70 & 0.8769 & 234.1 & 642.8 & 18.9 & 0.6375 \\
\hline 1000 & 1.00 & 1.52 & 15.0 & 6.80 & 65 & 0.8148 & 658.9 & 155.9 & 9.1 & 0.1529 \\
\hline 2000 & 0.50 & 1.50 & 16.0 & 12.00 & 70 & 0.7496 & $2098 . i$ & - & 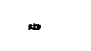 & - \\
\hline
\end{tabular}

above results; this makes them remarkable. The devices seem to operate best at frequencies below a faw gigahertz.

Several problems have been neglectedi; most important are analysis of the possible bending systems, analysis of beam effects on the circuit loading, and space charge in the output region. The first problem is straightforward; the author is programming the equations for a magnetic bending system. The second problem should not be difficult; a generalization of Rowe's metiods, ${ }^{11}$ in which the circuit equations are derived to relate the circuit wave amplitude to an induced current integral, can be used. This method is used often in traveling-wave $t$ e analysis. The space-charge problem is the most difficult because the electron beam is almost halted in t.te output region. The conventional Green's-function methods in Refs. 10 and 11 lose validity in these conditions.

The author has worked extensively on the optimization of klystrons and traveling-wave amplifier efficiency. Very rarely, if all parameters are optimized carefully, one can calculate an electronic efriciency of over $70 \%$ for a klystron. For the gyrocon, it is easy to find combinations of parameters that predict $>80 \%$ overall efficiency. Because the output circuit is a resonant ring, the gyrocon bandwidth will be narrow, but adequate for accelerators. Thus, the author believes that the gyrocon will find many applications where very efficient, high-power, low-frequency microwave amplifiers are required. 


\section{ACKNOWLEDGMIENTS}

The author is indebted to E. M. Rowe and F. E. Mills who indicated the general properties of the Russian invention. He also acknowledges the support and encouragement of T. J. Boyd, Jr, and D. A. Swenson.

\section{AEFERENCES}

1. J. W. McRae, "Harmonic Generation System," U.S. Patent No. 2408 437, October 1946.

2. G. 1. Budker et al., "The RF System of the VEPP-4 Electron-Positron Storage Ring Based on the Gyrocon - A. High-Power UHF Generator with an Unbunched Relativistic Beam," Proc. Dubna Accelerator Conf., Dubna, U.S.S.R., October 1976, to be published.

3. I. Kaufman and G. Oltman, "Harmonic Generation by Electron Beam Pattern Motion - the Bermutron," IEEE Trans. Electiun Devices ED-12, 31-¿9 (1965).

4. P. B. WilBon, "Notes on Trajectory-Modulated Electron Devices," Microwave Laboratnry report 527, W. Hansen Laboraicory of Physics, Stanfo d University (June 1956).

5. S. Ramo, J. R. Whinnery, and T. Van Duzen, Fields and Waves in Communication Eiscironics (John Wiley \& Sons, Inc., New York, 1965), pp. 421-429.

6. N. Marcuvitz, Waveguide Handbook (Dover Publications, New York, 1951), pp. 397-398.

7. W. K. H. Panofsky and W. A. Wenzę, "Some Considerations Concerning the Transverse Deflection of Charged Particles in Radio-Frequency Fields," Rev. Sci. Instrum. 27, 967 (1956).

8. J. Haimson, "Optimization Criteria for Standing Wave Transverse Magnetic Deflection Cavities, "Prcc. 1966 Linear Accelerator Conf., October 1966, Lcs Alamos Scientific Laboratory report LA-2609, pp. 303331 (December 1966).

9. R. H. Miller, Stanford Linear Accelerator Center, private communiration, May 1977.

10. P. J. Tallerico and J. E. Rowe, "Relativistic Effects in the Traveling-Wave Amplifier," IEEE Trans. on Electron Devices ED-17, 549-561 (1970).

11. J. E. Rowe, Nonlinear Electron-Wave Interaction Phenomena (Academic Press Inc, New York, 1965), pp. 28-62. 


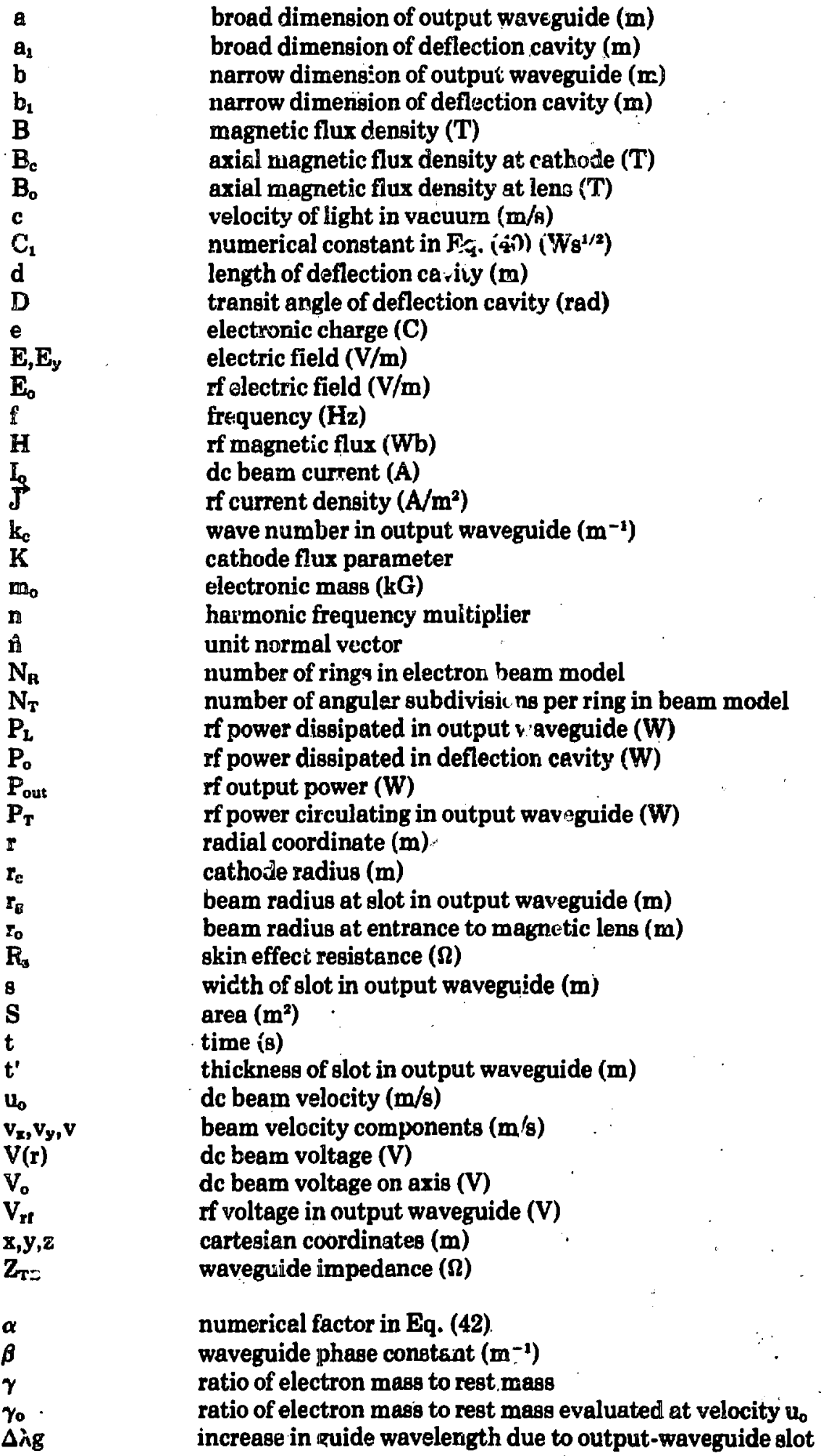


permitivity of free space $(\mathrm{F} / \mathrm{m})$

electronic charge-to-mass ratio ( $\mathrm{C} / \mathrm{kG}$ )

overall dc-to-rf energy conversion efficiency

output circuit efficiency

deflection circuit efficiency

electronic conversion efficiency

focus and bending system efficiency

characteristic impedance of free space $(\Omega)$

free-space wavelength (m)

guide wu. elengih in output waveguide (m)

permeability of free spracs $(\mathrm{H} / \mathrm{m})$

normalized entrance position of beam into output waveguide

conductivity of a conductor $\left(\mathrm{m}^{-1} \mathrm{a}^{-1}\right)$

transit time of electron (B)

phase angle (rad)

deflection angle (rad)

radian frequency ( $\mathrm{rad} / \mathrm{s})$ 